\title{
Parton energy loss in the reformulated weakly-coupled kinetic approach
}

\section{Tianyu Dai*}

Department of Physics, Duke University

Durham, NC27708, USA

E-mail: tianyu.dai@duke.edu

\section{Steffen A. Bass}

Department of Physics, Duke University

Durham, NC27708, USA

E-mail: bass@phy . duke.edu

\section{Jean-François Paquet}

Department of Physics, Duke University

Durham, NC27708, USA

E-mail: jeanfrancois.paquet dduke.edu

\section{Derek Teaney}

Department of Physics \& Astronomy, Stony Brook University

Stony Brook, NY11764, USA

E-mail: derek.teaney@ stonybrook. edu

\begin{abstract}
Interactions between hard partons and the quark-gluon plasma range from frequent soft interactions to rare hard scatterings. The larger number of soft interactions makes possible an effective stochastic description of parton-plasma interactions in terms of drag and diffusion transport coefficients. In this work, we present a numerical implementation that builds upon this systematic division between soft and hard parton-plasma interactions. We study the dependence of the single parton distribution on the cutoff between soft and hard parton-plasma interactions, both for small and phenomenological values of the strong coupling constant.
\end{abstract}

International Conference on Hard and Electromagnetic Probes of High-Energy Nuclear Collisions 30 September - 5 October 2018

Aix-Les-Bains, Savoie, France

${ }^{*}$ Speaker. 


\section{Introduction}

The energy loss of a hard parton traveling in the quark-gluon plasma can be studied within a weakly-coupled kinetic approach. Parton-plasma interactions is treated perturbatively, and the dynamics of well-defined quasiparticles (quarks and gluons) are described by transport equations [1]. Both the energy gain and loss of the partons are included naturally.

Leading-order realizations of weakly-coupled effective kinetic theory, such as MARTINI [2], generally divide parton-plasma interactions as elastic and inelastic processes. In Ref. [3], it was shown that the parton energy loss can be equivalently reformulated in terms of hard large-angle interactions and soft small-angle collisions. A major advantage of this reformulated approach is that it could be extended to next-to-leading order $[4,3]$. There are nevertheless important benefits at leading order as well. Soft and hard parton-plasma interactions can be systematically factorized. The large number of soft interactions can be efficiently described in a stochastic approach with transport coefficients; these drag and diffusion coefficients absorb plasma effects (e.g. Debye screening) that are particularly important for soft interactions. Large-angle interactions can be treated separately with emission rates, as in previous implementations.

In this work, we present the first numerical implementation of this reformulated energy loss model. We study the dependence of the parton energy loss on the cutoffs dividing the soft and hard interactions. We show that the reformulated energy loss remains valid at large values of the strong coupling constant $\alpha_{s}$.

\section{Description of the Reformulated Model}

The Boltzmann transport equation for a parton propagating through the quark-gluon plasma is $p \cdot \partial f=-(p \cdot u) \mathscr{C}[f]$, where $p$ is its four-momentum, $f$ is the distribution of partons, $u$ is the velocity of the medium and $\mathscr{C}[f]$ is the collision kernel. In previous implementations, $\mathscr{C}[f]$ would be divided into an elastic and an inelastic term. In the leading order reformulation [3], the collision kernel is divided into (i) hard elastic and inelastic interactions, (ii) diffusion, and (iii) conversion processes.

Hard interactions In the case of inelastic interactions, multiple soft interactions with the plasma induce the radiation of a parton of energy $\omega$. These induced parton emissions can be divided as large- $\omega$ and small- $\omega$ interactions by a cutoff $\mu_{\omega}$ with $\mu_{\omega} \lesssim T$, where $T$ is the temperature of the plasma. Small- $\omega$ inelastic interactions are absorbed into drag and diffusion coefficients. Large- $\omega$ inelastic interactions are described with emission rates, which are obtained from the AMY integral equation [5].

In the elastic case, a kinematic cutoff is imposed on the transverse momentum transfer $q_{\perp}$. The cutoff $\mu_{q_{\perp}}$ is chosen such that $g T \ll \mu_{q_{\perp}} \ll T$, with $g=\sqrt{4 \pi \alpha_{s}}$. Small- $q_{\perp}$ interactions are again absorbed into transport coefficients. Large- $q_{\perp}$ interactions are calculated perturbatively. Because plasma effects are significant only at low $q_{\perp}$, it is sufficient to use vacuum matrix elements to compute the large- $q_{\perp}$ rate. Given that $p \gg T$ is an excellent approximation for phenomenological applications, we further simplify the evaluation of the large- $q_{\perp}$ rate by keeping only the zerothorder term in $T / p$. 
Drag and diffusion Number- and identity-preserving soft interactions are described stochastically with drag and diffusion. Elastic as well inelastic interactions are included: because soft radiated particles are absorbed by the plasma, the number-preserving assumption still holds in the inelastic case. The diffusion processes can be described by a Fokker-Planck equation:

$$
\mathscr{C}^{\text {diff }}[f]=-\frac{\partial}{\partial p^{i}}\left[\eta_{D}(p) p^{i} f(\vec{p})\right]-\frac{1}{2} \frac{\partial^{2}}{\partial p^{i} \partial p^{j}}\left\{\left[\hat{p}^{i} \hat{p}^{j} \hat{q}_{L}(p)+\frac{1}{2}\left(\delta^{i j}-\hat{p}^{i} \hat{p}^{j}\right) \hat{q}(p)\right] f(\vec{p})\right\}
$$

where $\eta_{D}$ is the drag, and $\hat{q}_{L}(p)$ and $\hat{q}$ are the longitudinal and transverse momentum diffusion coefficients.

The latter are calculated perturbatively while the drag coefficient $\eta_{D}$ is obtained by the Einstein relation. The elastic contribution to both $\hat{q}$ and $\hat{q}_{L}$ can be found in Ref. [3]. Inelastic interactions also contribute to the longitudinal diffusion: $\hat{q}_{L}^{\text {inel }}=\frac{(2-\ln 2) g^{4} C_{R} C_{A} T^{2} \mu_{\omega}}{4 \pi^{3}}$.

Conversion An additional type of interactions are conversions, in which the incoming parton changes identity. The leading contribution to this process, both in the elastic and inelastic cases, is suppressed by $\mathscr{O}(T / p)$ compared to the large-angle and diffusion processes. Consequently, their contribution is not included in this work.

Reformulation The reformulation can be written as:

$$
\mathscr{C}=\mathscr{C}^{2 \leftrightarrow 2}+\mathscr{C}^{1 \leftrightarrow 2}=\mathscr{C}^{\text {large- } \omega}\left(\mu_{\omega}\right)+\mathscr{C}^{\text {large- } q_{\perp}}\left(\mu_{q_{\perp}}\right)+\mathscr{C}^{\text {diff }}\left(\mu_{\omega}, \mu_{q_{\perp}}\right)+\mathscr{C}^{\text {conv }}\left(\mu_{q_{\perp}}\right)
$$

Every term has a cutoff dependence, which cancels out when added together. In what follows we test this cutoff independence numerically, for the first time.

\section{Numerical implementation $\&$ results in a static medium}

To implement the reformulated energy loss model described in the previous section, we used the public version of the JETSCAPE framework [6], building upon the existing implementation of MARTINI [2] already in the framework. The Fokker-Planck equation describing the soft interactions (2.1) was added as a Langevin equation, solved with the pre-point Ito scheme.

Strictly speaking, the reformulation of parton energy loss Eq. (2.2) is valid in the small coupling limit. Independence on the cutoffs separating soft and hard interactions $\left(\mu_{q_{\perp}}\right.$ and $\left.\mu_{\omega}\right)$ is not assured in phenomenological applications, where a large coupling is used. We discuss this cutoff dependence in what follows. Because the cancellation of the cutoff is essentially independent in the elastic and inelastic cases, we look at them separately.

Inelastic energy loss We set $\alpha_{s}=0.3$ and simulate the propagation of a $20 \mathrm{GeV}$ gluon traveling for $1 \mathrm{fm} / \mathrm{c}$ in an infinite static medium. Only inelastic energy loss is included. The temperature of the plasma is $300 \mathrm{MeV}$. We vary the inelastic cutoff $\mu_{\omega}$ to the value $\mu_{\omega}=0.25 T, T, 2 T$. The leading-gluon energy distribution is shown in Figure 1, first separately for the large- $\omega$ interactions and the drag and diffusion, and then combined.

In the large- $\omega$ case (Figure 1(a)), all soft radiations below the cutoff $\mu_{\omega}$ are forbidden; inevitably, the energy distribution around the initial parton energy is found to depend on $\mu_{\omega}$. In the drag and diffusion case (Figure 1(b)), because the transport coefficients increase linearly with $\mu_{\omega}$, 


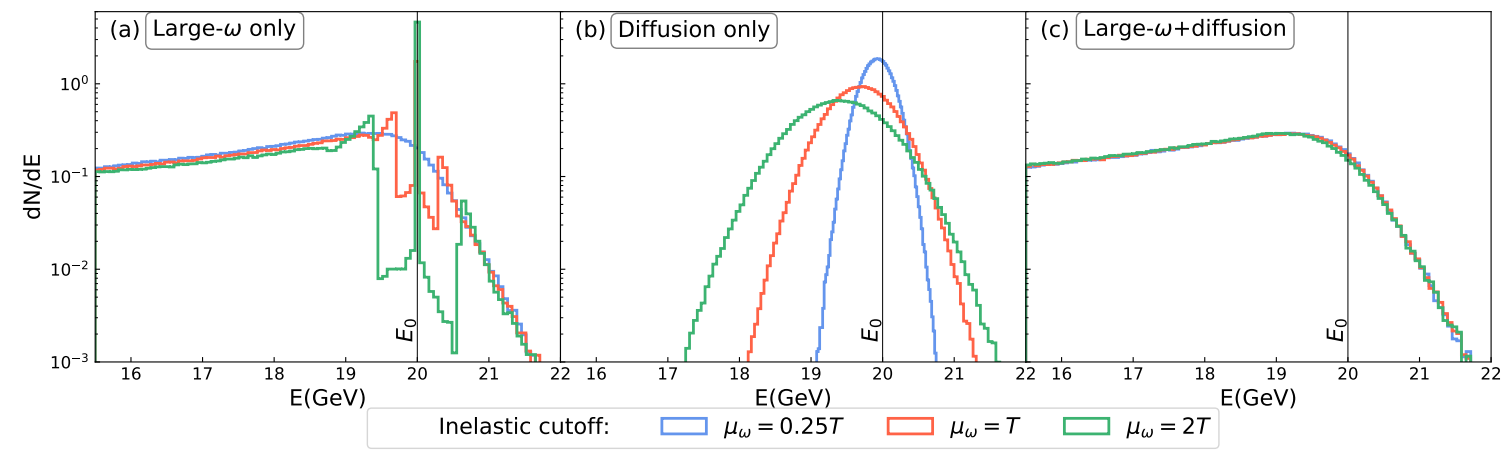

Figure 1: Energy distribution of a $20 \mathrm{GeV}$ gluon losing energy through inelastic processes only in a $1 \mathrm{fm} / \mathrm{c}$ static quark-gluon plasma at $\mathrm{T}=300 \mathrm{MeV}$, for different $\mu_{\omega}$. For (a) large- $\omega$ only, (b) small- $\omega$ only, and (c) combined energy loss.

the parton energy loss also increases on $\mu_{\omega}$. Once combined, Figure 1(c), the $\mu_{\omega}$ dependence of the parton energy loss cancels out.

In fact, when $\mu_{\omega}$ is decreased, we reach the limit where the effect of small- $\omega$ radiation is negligible. This is the limit in which inelastic energy loss is typically implemented. The reformulated energy loss allows for this cutoff to be increased and varied.

We verified that results similar to Figure 1 were obtained with (i) a smaller coupling constant, (ii) different initial parton energies, (iii) a quark propagating instead of a gluon, and (iv) a realistic hydrodynamic medium used instead of a brick.

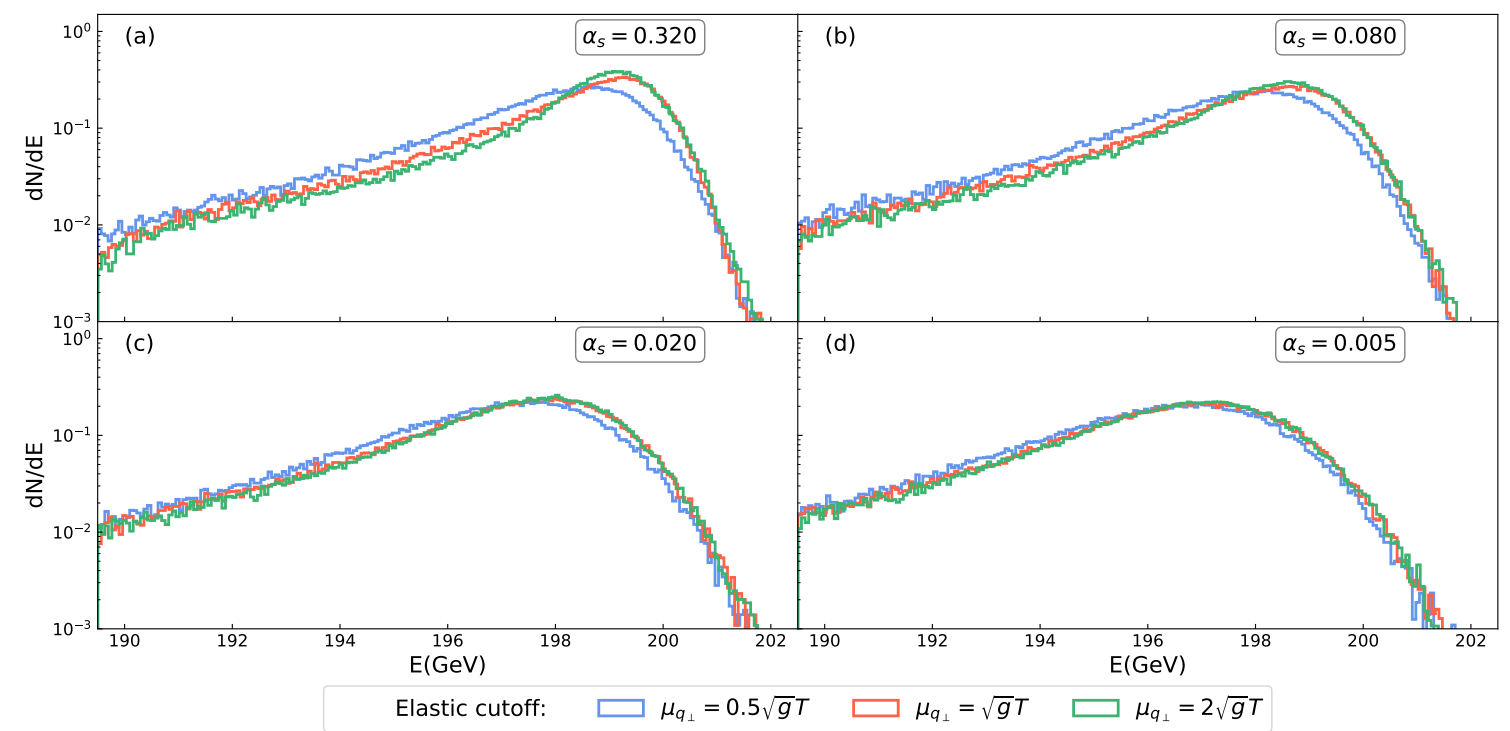

Figure 2: Energy distribution of a gluon with initial energy $E_{0}=200 \mathrm{GeV}$ losing energy through elastic processes in a static plasma with $T=300 \mathrm{MeV}$, for a propagation length $\tau=\alpha_{s}^{-2} 0.3^{2} \mathrm{fm} / \mathrm{c}$ at different elastic cutoff $\mu_{q_{\perp}}$. The results are shown for four different coupling constants $\alpha_{s}$.

Elastic energy loss Figure 2(a) shows the energy distribution of a $200 \mathrm{GeV}$ gluon propagating for $1 \mathrm{fm} / \mathrm{c}$ in the same medium as used in the previous example: infinite length, $T=300 \mathrm{MeV}$. 
Only elastic energy loss is included. We show only the combined result with both large-angle scattering and drag and diffusion. At large coupling, the dependence on the elastic cutoff $\mu_{q_{\perp}}$ is modest, although larger than found in the inelastic case. This larger cutoff dependence, in the large coupling limit, is likely a consequence of our use of vacuum matrix elements in the large $q_{\perp}$ elastic energy loss calculation, instead of screened matrix elements. In the small coupling regime, screening effects are small for large- $q_{\perp}$ interactions, and the use of vacuum matrix elements is enough. As expected, we find that the cutoff independence is recovered when the coupling is reduced $^{1}$, as shown in Figure 2(b-c).

\section{Summary \& outlook}

The reformulated parton energy loss from Ref. [3] provides a systematic factorization of soft and hard interactions in the weakly-coupled limit. The numerical implementation presented in this work indicates that this factorization still holds well at large coupling and can be used in phenomenological studies.

Naturally one benefit of this reformulation is the possibility of extending it to next-to-leading order [3]. A separate benefit is phenomenological: this methodical separation of the transport (soft) sector of the parton energy loss from hard interactions provides a framework for data-driven constraints on the drag and diffusion coefficient of partons. We expect both of these directions to have important applications for the study of jets in heavy ion collisions.

Acknowledgements We are grateful to Jacopo Ghiglieri, Weiyao Ke and Yingru Xu for their help with this project. We thank Bjoern Schenke and Heikki Mäntysaari for their collaboration in the early stage of this project, and the JETSCAPE Collaboration for their assistance with the JETSCAPE framework. This work was supported by the National Science Foundation under Award Number ACI-1550225 (T.D.), the U.S. Department of Energy under Award Numbers DE-FG0205ER41367 (S.A.B., T.D., J.-F.P.) and DE-FG02-88ER40388 (J.-F.P., D.T.).

\section{References}

[1] P. B. Arnold, G. D. Moore and L. G. Yaffe, Effective kinetic theory for high temperature gauge theories, Journal of High Energy Physics 2003 (2003) 030.

[2] B. Schenke, C. Gale and S. Jeon, MARTINI: An Event generator for relativistic heavy-ion collisions, Phys. Rev. C80 (2009) 054913 [0909.2037].

[3] J. Ghiglieri, G. D. Moore and D. Teaney, Jet-medium interactions at nlo in a weakly-coupled quark-gluon plasma, Journal of High Energy Physics 2016 (2016) 95.

[4] S. Caron-Huot, $O(g$ ) plasma effects in jet quenching, Physical Review D 79 (2009) 065039.

[5] P. B. Arnold, G. D. Moore and L. G. Yaffe, Photon and gluon emission in relativistic plasmas, JHEP 06 (2002) 030 [hep-ph/ 0204343$].$

[6] K. Kauder, Jetscape v1. 0 quickstart guide, arXiv preprint arXiv:1807.09615 (2018).

\footnotetext{
${ }^{1}$ We fix the propagation length $\tau=\alpha_{s}^{-2} 0.3^{2} \mathrm{fm} / \mathrm{c}$ to make the number of elastic collisions the same for tests with different coupling constants.
} 